\title{
Heat exhaust in fusion reactors and its impact on the sustainability of fusion electricity
}

\author{
Giacomo Dose and Simone Noce \\ University of Rome "Tor Vergata", Department of Industrial Engineering \\ Via del Politecnico 1, 00133 , Rome, Italy
}

\begin{abstract}
Summary. - This contribution presents a didactic overview of the sustainability of nuclear fusion as a source for electricity production. A comparison with traditional means of electricity generation will be carried out, especially regarding waste production. Particular attention will be focused on the lifetime of components in a fusion reactor, since this engineering parameter determines directly the environmental and economic sustainability. Finally, the impact of heat exhaust components, which is the field of expertise of the authors, on waste production will be assessed and the most active research activities aimed to enhance the performance and lifetime of these components will be summarized.
\end{abstract}

\section{1. - Waste production in a fusion reactor}

Presently, the world is more than ever concerned in achieving sustainable energy production. Estimates by the United Nations indicate that in the next 30 years more than 10 billion people will be living on our Planet [1], and providing to all of them the same energy consumption of the present developed world represents a great challenge. It is not clear whether human kind will be able to provide this vast amount of power by 
sticking to the current energy mix, but without doubt increasing energy production while relying heavily onto fossil fuels will lead to serious environmental and health problems [2]. A shift is mandatory towards a sustainable energy future. A fundamental role will be played by improvements in energy efficiency, and advancements in technology will surely allow to generate a greater amount of power starting from the same amount resources. However, this alone cannot solve the issue. It is essential to make a transition towards less resource-intensive energy sources, able to consume a minimal amount of material and fuel and therefore producing the smallest possible amount of waste per TWh of delivered energy. One often forgets that waste generation is an intrinsic part of any conversion process. Thermodynamics is very clear about that; there is no free meal. Whatever the energy source, waste will always be generated. This not only covers the waste from the power production process itself, but also includes all the material to be disposed of at the end-of-life of the system. From this point of view, nuclear energy represents the most suitable candidate to satisfy our future energy, and in particular our electricity needs. This source offers by far the lowest material consumption per TWh of electricity produced [3] and without emission of greenhouse gases. Nuclear fusion, in particular, has advantages that ensure the sustainability and security of supply: fuel is virtually unlimited and available, no long-lived radioactive waste is produced and it is intrinsically safe since a chain reaction is physically impossible inside a fusion reactor.

But, in what consists the waste of nuclear fusion?

Let us begin evaluating the waste that is generated from the fusion reaction alone. The nuclear process that is now pursued is the deuterium-tritium fusion reaction. From each reaction a helium-4 nucleus and a fast neutron are produced. The first is pumped away from the reactor and the latter is absorbed either by the wall components or a section of the reactor called the "breeder", containing lithium that is split due to the bombardment with the high energy neutrons. As a result, tritium nuclei are generated. In fact, tritium is not available on Earth, since it is a radioisotope with a half-life of 12.3 years, and must thus be produced ad hoc. As a result of this breeding reaction, another helium-4 nucleus and a slow neutron are produced. The latter either shares the same destiny of its predecessor or is absorbed by other solid parts of the reactor. However, in order to obtain tritium self-sufficiency, we must produce $\sim 15 \%$ more tritium than the amount burned in the reactor, in order to compensate losses and provide storage for future needs (e.g., tritium to start a new reactor, manage possible failures of the fuel cycle system). In conclusion, considering the reactor as a black-box, deuterium and lithium are burned, and helium- 4 is produced. A reactor generating $1 \mathrm{GW}_{\mathrm{th}}$ of fusion power during one year would generate $159.8 \mathrm{~kg}$ of helium-4, of which $74.3 \mathrm{~kg}$ due to the fusion reaction itself and $85.5 \mathrm{~kg}$ to tritium breeding, by consuming $37.4 \mathrm{~kg}$ of Deuterium and $113.6 \mathrm{~kg}$ of lithium (90\% enriched in lithium-6). A vast amount of power is thus generated by using a small amount of fuel. Regarding the fusion reaction, the only waste is helium-4, which is not toxic, is not a greenhouse gas and only a few hundred kg per GWyear $_{\text {th }}$ are produced. By comparison, the same thermal energy could be obtained by burning more than 1 billion $\mathrm{kg}$ of coal (or 0.5 billion $\mathrm{kg}$ of natural gas), releasing more than 3 billion $\mathrm{kg}$ ( or 1.5 billion $\mathrm{kg}$ ) of $\mathrm{CO}_{2}$ in the atmosphere. 
Therefore, nuclear fusion offers, as far as the waste from the reaction is concerned, a sustainable prospect for electricity production. However, as we explained before, waste does not include only the products of the reaction but also all the material to be disposed of at the end-of-life of the system. This waste volume is tightly linked to the lifetime of the components inside the reactor. The flux of particles, photons and, most of all, neutrons leads to damage of the solid walls closest to the fusion reactions, determining the lifetime of all parts inside the reactor. Unfortunately, the present technology is not capable of providing components that all have the same lifetime, and therefore damaged parts of the device must be replaced at regular time intervals over the lifetime of the reactor. The need to change some components during the lifetime of the plant obviously implies an increase in material use and must be also included when accounting for the total waste production. In [4] a detailed analysis has been carried out for the DEMO reactor, the European fusion plant currently under design that should supply electricity to the grid in the second half of this century. A total of sixty thousand tonnes of waste are expected to be produced over the whole twenty-three years of operation of the plant. Normalizing this quantity to the "reference energy" we used previously $\left(1 \mathrm{GW}_{\text {th }}\right.$ continuously for a year), one obtains five thousand tonnes of waste. In fact, due to the lifetime of the structural components under neutron irradiation, DEMO is limited to produce $12 \mathrm{GWyear}_{\mathrm{th}}$, resulting from $2 \mathrm{GW}_{\text {th }}$ produced in 6 full-power years. Note that no long-lived radioactive waste is produced by fusion, and the entirety of this mass is made of either low or intermediate level waste [4]. This represents one of the main advantages of fusion: no geological disposal is needed and almost all the waste material is potentially recyclable after 100-200 years (when the contact dose rate has decayed under the recycling limit).

The major contribution to the waste is therefore due to the materials to be disposed of at the end-of-life of the system; 5 million $\mathrm{kg} v s .160 \mathrm{~kg}$ from the reaction alone per GWyear $_{\text {th }}$. A comparison with the waste produced by traditional sources of power generation is more difficult to carry out, since the estimate of the waste material to be disposed of at the end-of-life of the system would require an in-depth analysis which is outside the scope of this contribution. However, even neglecting this relevant contribution, the substantial difference between the orders of magnitude of the mass of waste produced that we calculated up to now (millions of $\mathrm{kg} v s$. billions of $\mathrm{kg}$ ), should leave no doubts on the sustainability of fusion energy for electricity production. Note also that less waste has a twofold contribution to sustainability; reduced disposal, and reduced consumption of resources. Moreover, there is still room for additional improvements, since a substantial part of the waste is not intrinsically linked to the reaction, as in the case of fossil fuels, but it is linked to the present technological capabilities. The component lifetime then represents the key parameter determining the sustainability of fusion energy and improving the lifetime of the components is in fact one of the most active fields of research regarding the components inside a fusion reactor. In the next sections, the focus will be turned to the main factors that affect this engineering parameter, and in particular regarding the parts of the reactor that are closest to the hot plasma where the fusion reactions take place, usually referred as plasma-facing components (PFCs). 
TABLE I. - Differences between the different nature of loading inside a fusion reactor.

\begin{tabular}{l|cccl}
\hline & \multicolumn{2}{|c}{ Deposition } & Power density & Other interactions \\
\hline Photons & Distributed & On Surface & $0.5 \mathrm{MWm}^{-2}$ & Negligible \\
\hline Charged particles & Local & On Surface & tens of $\mathrm{MWm}^{-2}$ & Sputtering \\
\hline Neutrons & Distributed & On Volume & Negligible & Dpa \\
& & & & He production \\
& & & Trasmutations \\
& & & & Activation \\
\hline
\end{tabular}

\section{2. - Lifetime of components that exhaust heat inside a fusion reactor}

We discussed about the sustainability of nuclear fusion, and how this is determined by the lifetime of components. In simple terms, the longer a part can be kept inside the device, the less waste it will contribute to the total waste associated to the energy production.

However, what determines the lifetime of components in a fusion reactor?

To answer this question, one must firstly understand the loads on the components inside a fusion machine. Loads are caused mainly by photons, generated both from the deflection or deceleration of charged particles and from the change in the energy state of bound electrons in neutral or partially ionized atoms; there is also a contribution from charged particles that deposit their kinetic energy on the PFCs where the magnetic field lines intersect the solid walls; finally high-energy neutrons from the deuterium-tritium reaction cause also damage, since they are not confined by the magnetic field and directly bombard the wall. The engineering design of components inside a fusion reactor must take into account all of these effects, resulting in a fascinating multidisciplinary endeavor. The impact of the different nature of loading can be summarized evaluating three main aspects: the morphology of the deposition, the power density associated to it and the interaction (apart from energy deposition) between wall and impinging particles. This is summarized in table I. Let us remark that photons and neutrons are emitted isotropically, while charged particles are mainly deposited on the narrow area, where the magnetic field lines intersect the wall. Moreover, photons and particles interact only with the surface layer of the wall while neutrons penetrate deeply inside the material, some even escaping the reaction chamber and reaching the external bioshield. These differences in the deposition affect heavily the power density deposited, which determines directly the thermo-mechanical stresses inside the components. The power density depends both on the power carried by each "energy channel" (photons, particles or neutrons) and on the deposition volume. In fact, even if neutrons carry almost four fifths of the total thermal power of the reactor, their contribution to the thermo-mechanical stresses are negligible compared to the other two channels due to the fact that the energy is deposited basically on the whole volume of the wall materials. On the other hand, photons and particles are stopped at the surface and their deposited power density (now considered per 
unit surface, due to negligible penetration) is the major contribution to thermal stress. Photons and charged particles carry the remaining portion of the thermal power of the fusion device, which is the amount used to sustain the nuclear reactions, the so-called plasma heating power. While electromagnetic waves are emitted isotropically, resulting in a power density that for DEMO is expected to be $0.5 \mathrm{MWm}^{-2}$, particles are stopped mainly in local spots of the wall. In fact, the energy associated to the charged particles leaks out from the central part of the plasma chamber, flows through a millimeters-thick plasma cortex and reaches finally the so-called divertor. Due to the small exchange area at the divertor, the power density reaches values higher than $20 \mathrm{MWm}^{-2}$. To give an idea, this power density is of the same order of magnitude of the power density leaving the surface of the Sun, which is $\sim 60 \mathrm{MWm}^{-2}$. The thermal stresses generated from such power densities can lead to plastic deformation, which causes the cracking of the surface of the wall materials due to low cycle fatigue failure. In addition, the pulsed nature of a fusion reactor results in cyclic loads, leading to a phenomenon called Creep-Fatigue which consists in the deformation of a material under repeated stresses at high temperature. This also influences the component lifetime. In addition, particles do not only deposit energy but also interact with the walls in various ways. Charged particles collide with atoms of the target material, and exchange momentum. This could lead to the ejection of atoms from the solid surface, through a process called sputtering. This form of erosion depends on the material used. For this reason, the entire wall of the reaction chamber is made of tungsten $(\mathrm{W})$, which has a high threshold to sputtering. In particular, the divertor targets, where the particle flux is expected to be the highest, are provided with the highest thickness of W. The interaction of neutrons is, instead, of nuclear nature and depends therefore on the neutron energy spectrum, the fluence and the elements of the periodic table used for the wall material. Neutrons can damage the wall in many ways. First, nuclear reactions with the atomic nuclei can occur, leading to transmutation (the production of other elements which have different properties than the original ones), production of light gas such as hydrogen and, most of all, helium inside the lattice material (which leads to swelling and cluster formation and worsens the mechanical properties) and radioactivity (which can be the result of transmutations or other nuclear processes and represents an important safety issue). Moreover, when high-energy neutrons bombard a solid material, they can displace nuclei inside the lattice. This results in a redistribution of the atoms of the material and can occur several times inside the lattice. This phenomenon, referred as neutron irradiation damage, is measured with a quantity called "displacement per atom" (or dpa) and is defined as the number of times that an atom is displaced, on average, for a given fluence. Among the various types of damage that we listed, this is at present time the main limiting factor to component lifetime. While the other damages are focused on the parts closest to the plasma where the fusion reactions take place, the neutron irradiation damage affects also the structural components, even if they are rather far from the burning plasma. As an example, the EUROFER structural steel has a limit of 50 dpa over the lifetime of the component, which can be reached in just few years in certain parts of the reactor. Therefore, a proper material choice and the development of innovative solutions are key focus points in fusion research. 
TABLE II. - Waste produced in DEMO over the lifetime and per GWyear th $_{\text {generated. }}$

\begin{tabular}{c|cc}
\hline & Whole Plant & Amount due to PFCs \\
\hline Over the lifetime & 60000 tonnes & 1773 tonnes \\
Per GWyear th $_{\text {th }}$ & 5000 tonnes & 147.7 tonnes \\
\hline
\end{tabular}

Even if the amount of dpa is the main limiting factor to the lifetime of the structural components, the lifetime of PFCs is also affected from erosion due to sputtering and the thermal loads. Besides the divertor, other PFCs exist inside a fusion device, namely limiters and first wall. The firsts are located only in some discrete parts of the reaction chamber and are used for transient phases, where the plasma is in contact with the wall, while the latter can be defined as the component that covers the remaining plasmafacing surface. Considering all PFCs, they account for 1773 of the total 60000 tonnes of waste produced during the whole operating life of DEMO, and of the 5 million $\mathrm{kg}$ per $\mathrm{GW}_{\text {th }}$ per year mentioned before they account for $147753 \mathrm{~kg}$ (table II). Therefore, the components that exhaust heat inside a fusion reactor represent $3 \%$ of the total generated waste, and their impact on the overall mass is marginal. However note that, due to their position in the reactor, the PFCs receive the highest neutron irradiation, thus having the highest level of activation. Therefore, reducing this portion of the waste would impact directly the amount with the highest radioactivity, especially taking into account that PFCs are largely made of $\mathrm{W}$, which is an element prone to activation (meaning that it easily produces radioisotopes when irradiated with neutrons). Increasing the PFC lifetime would then target the most critical fraction of the waste, with benefits to both safety and waste management.

\section{3. - How can we improve lifetime of PFCs and the current active research?}

All actively cooled PFCs are provided with an armor, the plasma-facing part of the component, and a heat sink, which is the part that hosts the cooling channels and usually performs the structural function. The material of the armor, as well as its thickness, are chosen in order to take into account sputtering and other aspects influencing the compatibility with the plasma. Where the particle flux is the highest, the thickness of the $\mathrm{W}$ layer will be increased so that erosion would not affect substantially the lifetime. However, damage to thermal fatigue and thermal shocks can induce cracking on the plasma-facing surface, which could compromise the PFC integrity. As for the choice of the materials for the heat sink, various constraints have to be taken into account that are important for the exhaust capability and the structural integrity. The thermal conductivity must be maximized, as for all steady-state heat exchangers, in order to decrease the temperature gradient inside the material which affects directly the thermal stresses and the maximum surface temperature. Thermal stresses are also dependent on the coefficient of thermal expansion (CTE), as well as the ratio between the CTE of the armor and that of the heat sink material. In fact, one of the most critical interfaces in PFCs is that between W 
and the heat sink, where stresses intensify, since the armor does not experience the same magnitude of thermal expansion as the heat sink. Therefore, $\mathrm{W}$ undergoes a tensile stress whereas the heat sink undergoes a compression. Lastly, ductility is a key property for materials used in structural parts, and it consists in the capability of undergoing deformation before rupture or breaking. Loss of ductility, also called embrittlement, can occur after neutron damage. The resistance to neutron embrittlement is therefore a key property for the choice of a heat sink material. As the amount of dpa depends on neutron fluence, the less a material is prone to embrittlement the longer is the lifetime inside the reactor.

How are these various problems tackled in fusion research?

Plasma-facing material. Avoiding cracks induced by thermal fatigue or thermal shocks is the central objective of this research. A potential candidate can be found in liquid metals [5], which are able to "self-heal" thanks to the refilling of the surface. Moreover, a liquid would not generate any thermal stress at the interface with the heat sink. Main engineering challenges consist in corrosion management, the confinement of the liquid against the electromechanical forces and the refilling. Other solutions proposed concern the use of structured W [6] instead of bulk material. Having a structured geometry, the material is able to freely expand, without generating stresses. Moreover, latticed W could also be used in sacrificial limiters [7] providing protection to the machine during off-nominal events, since it could potentially mitigate damage by favoring vapor shielding. The main challenges are linked to quality assessment and manufacturing of large component areas (hundreds of $\mathrm{m}^{2}$ needed for a reactor), as well as the stability of the geometry during operation. Another approach used by the materials science community to address this issue is to add some ductility (or pseudo ductility) by alloying tungsten with other elements [8], by using W-fibre reinforced composite materials [9] or by controlling its granular structure. At present, these developments are however in the beginning stages, and the technology is not yet mature to manufacture components.

Heat sink material. Resistance to neutron embrittlement is the key focus for this research. State-of-the-art PFCs contain a heat sink made of a copper alloy ( $\mathrm{CuCrZr}$ ). However, the mechanical properties of $\mathrm{CuCrZr}$ degrade even at around $10 \mathrm{dpa}$, which forbids its use for PFCs with the highest neutron fluence. For the first wall, Reduced Activation Ferritic/Martensitic (RAFM) steels represent one of the potential alternatives, leading to the development of novel steel-based designs of PFCs [10]. The engineering challenge consists in managing the increased thermal stresses that arise from the low thermal conductivity of RAFM steels, which is 10-15 times lower than that of $\mathrm{Cu}$. W-based composite materials are also actively investigated, such as $\mathrm{W} / \mathrm{Cu}$ composite materials for the divertor [11], whose high heat fluxes exclude the possibility of using an insulating material such as steel, and W/Fe composites. Indeed, W provides a good resistance to neutron embrittlement, due to the high number of protons in the nucleus. However, no present experiment shares the same energy spectrum and neutron fluence of DEMO, and experimental validation for such novel materials is delayed until after the construction of a proper neutron source, like, e.g., IFMIF-DONES, which is planned to be realized before 2030 [12]. 
Manufacturing technology. As novel materials and new concepts are being developed, technology must be advanced accordingly to deliver a complete component. Particular attention is focused in the bonding process between the armor, usually made of tungsten, and the heat sink material, typically containing steel or copper. As mentioned before, the CTE mismatch between tungsten and the heat sink material intesifies thr stresses at the interface. A solution could consist in, Functionally Graded Materials (FGM), which are engineered composites characterized by a gradual variation of the composition over the material volume. Over the thickness, a smooth transition from a $100 \%$ to $0 \% \mathrm{~W}$ volume fraction is achieved, eliminating the abrupt discontinuity in the thermo-mechanical properties inside the material and reducing the stresses. Both FGM made of W/Fe [13], for the first wall, and $\mathrm{W} / \mathrm{Cu}[14,15]$, for the divertor, are being developed. The integration of a solution in a component is currently being tested. Main challenges consist in the increased complexity of the joining process compared to traditional solutions (diffusion bonding or vacuum brazing).

Finally, it should be mentioned that lifetime of PFCs is addressed not only by the engineering of the components, but also by acting on the plasma, with the aim to reduce the thermal loads. One way is to redirect some of the thermal power from the charged particle channel to the photon channel, effectively distributing the heat on a larger area. Another way is to increase the heat exchange area either by moving the divertor to larger radii (considering that a fusion reactor has a cylindrical geometry), increasing the number of divertor targets or making the magnetic field lines flare when they approach the wall. These are referred as advanced configurations, and are currently tested or planned in major fusion experiments around the world.

\section{REFERENCES}

[1] United Nations, World Population Prospects 2019: Highlights, https://population.un. org/wpp/Publications/Files/WPP2019_Highlights.pdf.

[2] Lelievelda J. et al., Proc. Natl. Acad. Sci. U.S.A., 116 (2019) 7192.

[3] Vidal E., Mineral Resources and Energy (Elsevier) 2017, ISBN: 9781785482670.

[4] Gilbert M. R. et al., Nucl. Fusion, 57 (2017) 046015.

[5] Roccella S. et al., "CPS based liquid metal Divertor Target for EU-DEMO", J. Fusion Energy, https://doi.org/10.1007/s10894-020-00263-4.

[6] Terra A. et al., Phys. Scr., T171 (2020) 014045.

[7] DE Luca R. et al., Fusion Eng. Des., 158 (2020) 111721.

[8] BrezinseK S. et al., Nucl. Fusion, 57 (2017) 116041.

[9] Linsmeier Ch. et al., Nucl. Fusion, 57 (2017) 092007.

[10] DTT Facility Team, Interim Design Report (Green Book), Chapt. 4, https://www . dtt-project.enea.it/downloads/DTT_IDR_2019_WEB.pdf.

[11] Müller A. v. et al., Phys. Scr., T171 (2020) 014015.

[12] Donné T. et al., European Research Roadmap to the Realisation of Fusion Energy (EUROfusion) 2018, ISBN 978-3-00-061152-0. 2018.

[13] Heuer S. et al., Phys. Scr., T171 (2020) 014028.

[14] Richou M. et al., Fusion Eng. Des., 157 (2020) 111610.

[15] Curzadd B. et al., Nucl. Fusion, 59 (2019) 086003. 